\title{
Distinct RNA N-demethylation pathways catalyzed by non-heme iron ALKBH5 and FTO enzymes enable regulation of formaldehyde release rates
}

\author{
Joel D.W. Toh ${ }^{a, 1}$, Steven W.M. Crossley ${ }^{a, 1}$, Kevin J. Bruemmer ${ }^{a}$, Eva J. Ge ${ }^{a}$, Dan $\mathrm{He}^{\mathrm{a}}$, \\ Diana A. Iovan ${ }^{\mathrm{a}}$ and Christopher J. Chang ${ }^{\mathrm{a}, \mathrm{b}, 2}$
}

a Department of Chemistry, University of California, Berkeley, Berkeley, CA 94720 USA

b Department of Molecular and Cell Biology, University of California, Berkeley, Berkeley, CA 94720 USA

1 Joel D.W. Toh and Steven W.M. Crossley contributed equally to this work

$2 \quad{ }^{*}$ Corresponding author: Christopher J. Chang, Email: chrischang@berkeley.edu

\section{Author ORCID}

Christopher J. Chang 0000-0001-5732-9497

Joel D.W. Toh 0000-0003-0253-2169

Steven W.M. Crossley 0000-0002-9932-3808

Kevin J. Bruemmer 0000-0003-3416-5615

Eva J. Ge 0000-0002-9238-1466

Dan He 0000-0002-9544-5672

Diana A. lovan 0000-0001-9889-7183

\section{Keywords}

non-heme iron enzyme, formaldehyde, m6A, one-carbon cycle, RNA epigenetics

\section{Author Contributions}

J.D.W.T., S.W.M.C., C.J.C. designed research; J.D.W.T., S.W.M.C., K.J.B., E.J.G., D.H., D.A.I. performed research; J.D.W.T., S.W.M.C., K.J.B., C.J.C. analyzed data; and J.D.W.T., S.W.M.C., C.J.C. wrote the paper with input from all authors.

This PDF file includes:

Main Text

Figures 1 to 6

Table of Contents Graphic

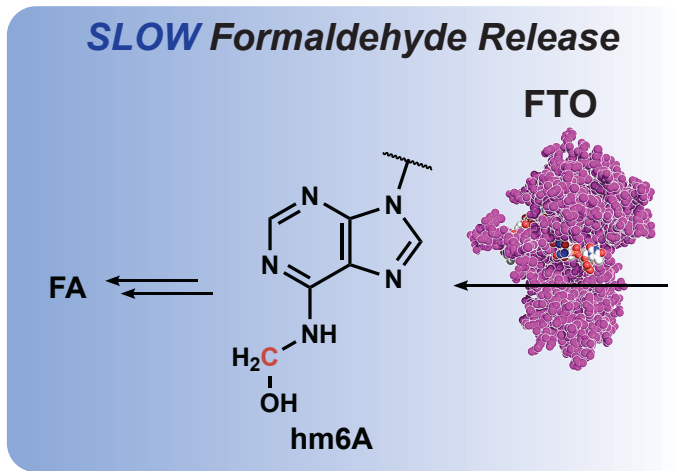<smiles>CCn1cnc2c(NC)ncnc21</smiles>

m6A
FAST Formaldehyde Release

ALKBH5

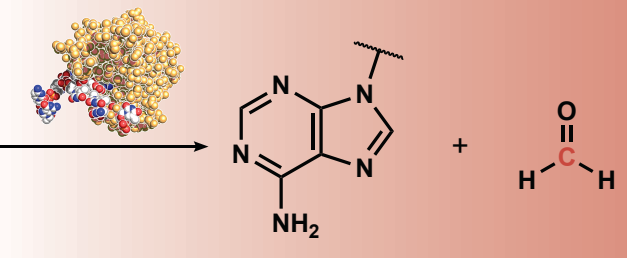

A 


\begin{abstract}
The AlkB family of non-heme-Fe(II)/2-oxoglutarate(2OG)-dependent oxygenases are essential regulators of RNA epigenetics by serving as erasers of one-carbon marks on RNA with release of formaldehyde (FA). Two major human AlkB family members, FTO and ALKBH5, both act as oxidative demethylases of N6-methyladenosine (m6A) but furnish different major products, N6hydroxymethyladenosine ( $\mathrm{hm} 6 \mathrm{~A})$ and adenosine $(\mathrm{A})$, respectively. Here we identify foundational mechanistic differences between FTO and ALKBH5 that promote these distinct biochemical outcomes. In contrast to $\mathrm{FTO}$, which follows a traditional oxidative $N$-demethylation pathway to catalyze conversion of $\mathrm{m} 6 \mathrm{~A}$ to $\mathrm{hm6A}$ with subsequent slow release of $\mathrm{A}$ and $\mathrm{FA}$, we find that ALKBH5 catalyzes a direct m6A-to-A transformation with rapid FA release. We identify a catalytic R130/K132/Y139 triad within ALKBH5 that facilitates release of FA via an unprecedented covalent-based demethylation mechanism with direct detection of a covalent intermediate. Importantly, a K132Q mutant furnishes an ALKBH5 enzyme with an m6A demethylation profile that resembles that of FTO, establishing the importance of this residue in the proposed covalent mechanism. Finally, we show that ALKBH5 is an endogenous source of FA in the cell by activity-based sensing of FA fluxes perturbed via ALKBH5 knockdown. This work provides a fundamental biochemical rationale for non-redundant roles of these RNA demethylases beyond different substrate preferences and cellular localization, where m6A demethylation by ALKBH5 versus FTO results in release of FA, an endogenous one-carbon unit but potential genotoxin, at different rates in living systems.
\end{abstract}

\title{
Significance Statement
}

Non-heme iron enzymes FTO and ALKBH5 play central roles in epigenetic RNA regulation by catalyzing the oxidation of N6-methyladenosine (m6A) to produce N6-hydroxymethyladenosine (hm6A) and adenosine (A), respectively. Here, we provide a mechanistic rationale for these distinct biochemical outcomes by identifying that ALKBH5 performs m6A demethylation via an unprecedented covalentbased mechanism with concomitant and rapid release of $A$ and formaldehyde (FA), whereas FTO liberates $\mathrm{hm} 6 \mathrm{~A}$ to release $\mathrm{A}$ and FA over longer timescales. This work reveals foundational biochemical differences between these closely related but non-redundant epigenetic enzymes and identifies ALKBH5 as an endogenous source of rapid formaldehyde generation in cells. 


\section{Main Text}

\section{Introduction}

The one-carbon (1-C) cycle is a universal hub in all living organisms that regulates processes spanning metabolism to epigenetics (1-6). Formaldehyde (FA) is a ubiquitous 1-C unit in this context with broad contributions to health and disease. Indeed, chronic exposure to elevated levels of FA is associated with various cancers and several neurological, diabetic, and cardiovascular diseases (6). However, FA is also endogenously produced by a diverse array of biochemical processes, such that substantial levels of FA are detectable in cells and animals (7-15), where it is metabolized by the ADH5 detoxification system (6). Included are pathways in folate-driven 1-C metabolism through serine hydromethyltransferase enzymes 1 and 2 (SHMT1 and SHMT2) to balance serine/glycine pools (2, 6, 16) as well as enzymes for epigenetic DNA and RNA regulation, such as non-heme-Fe(II)/2oxoglutarate(2OG)-dependent oxygenases (17-20) that serve as oxidative $N$-demethylases to erase one-carbon marks from nucleic acid substrates.

The JmjC $N^{\varepsilon}$-methyl-lysine demethylases (KDMS) and the AlkB family represent two major types of epigenetic enzymes $(20,21)$, where $N$-methyl oxidation of nucleic acid substrates results in FA release over a range of time scales depending on the stability of the resulting $N$-hydroxymethyl product (22). In particular, N6-hydroxymethyladenosine (hm6A) formed by mono-oxidation of m6A (23) is relatively stable compared to other hydroxymethyl-containing nucleobases and has been reported to decay to the free adenosine base over 10 hours (22). m6A is the most prominent modification of mRNA $(24,25)$ and is installed by the S-adenosylmethionine (SAM)-dependent METTL3/14-WTAP writer complex (26) and removed by two human AlkB eraser enzymes, fat mass and obesity-associated protein (FTO) (4) and AlkB family member 5 (ALKBH5) (27). Internal m6A modifications control the fate of mRNA (28-32) through translation, splicing, localization, stability, and decay and are connected to cancer progression, immune responses, and metabolic states $(27,29,32-36)$.

Despite their global similarities, the FTO and ALKBH5 RNA demethylases are non-redundant and exhibit key differences in RNA substrate preferences and cellular localization $(37,38)$. As such we became interested in deciphering other foundational features that define these epigenetic regulatory enzymes, particularly in the context of FA as both a genotoxin and endogenous mediator of the 1-C cycle. In this study, we now report the identification of distinct mechanistic pathways in FTO and ALKBH5 that promote differing timescales of FA release. Oxidative demethylation of $\mathrm{m} 6 \mathrm{~A}$ by FTO produces $\mathrm{hm6A}$ as the major product and we identify a role for E234 in stabilizing the nascent $\mathrm{hm} 6 \mathrm{~A}$ product, which slowly decomposes to A and FA, by analysis of the reported structure of the FTO:N6methyldeoxyadenosine (m6dA) complex (PDB ID 5ZMD) (39). In contrast, we find that ALKBH5 catalyzes a direct m6A-to-A conversion with concomitant rapid release of $F A$, undergoing a single nonheme $\mathrm{Fe}(\mathrm{II})-2 \mathrm{OG}-d e p e n d e n t$ oxidation and requiring one $2 \mathrm{OG}$ cofactor to produce one succinate molecule per m6A demethylation event. A key R130/K132/Y139 triad in the nucleotide binding region is responsible for the unique capacity of ALKBH5 to catalyze FA release, with direct detection of an unprecedented protein:substrate covalent intermediate that relies on K132 and $\mathrm{Y} 139$. Interestingly, a $\mathrm{K} 132 \mathrm{Q}$ ALKBH5 mutant abolishes the covalent-based mechanism and alters the m6A demethylation profile to resemble that of FTO. Finally, we show that ALKBH5 is an endogenous source of FA in cells by selective siRNA knockdown and activity-based sensing of this one-carbon unit. Taken together, our findings reveal a molecular basis for the divergent biochemical pathways of $\mathrm{N}$-demethylation and formaldehyde production catalyzed by FTO and ALKBH5, providing a starting point for further investigations into how and why epigenetic regulation is connected to 1-C metabolism through this dynamic one-carbon unit.

\section{Results and Discussion}

FTO and ALKBH5 Exhibit Distinct m6A Demethylation Profiles In Vitro. We first performed the characterization and quantification of the intermediates and products formed upon $\mathrm{m} 6 \mathrm{~A}$ demethylation catalyzed by FTO and ALKBH5. Quantification was performed by monitoring the unique mass fragment transition of respective nucleoside standards (SI Appendix, Fig. S1A-E), modified from reported methods $(23,40)$ using a UPLC-triple quadrupole LC-MS/MS. A and m6A nucleosides were commercially obtained (SI Appendix, Fig. S1A and B). hm6A was accessed by incubating A with $50 \mathrm{X}$ molar excess $F A$ overnight at $37^{\circ} \mathrm{C}$. hm6A was obtained by subtracting unreacted $A$ from known starting concentration of A standards (SI Appendix, Fig. S1C). f6A standard was synthesized in-house by modification of a reported procedure $(23,40)$ (SI Appendix, Fig. S1D). The enzymatic reactions were monitored at 1, 5, 10, 30 and 60 min. FTO $(5 \mu \mathrm{M})$ gave ca. $72 \% \mathrm{hm} 6 \mathrm{~A}, 6 \% \mathrm{f} 6 \mathrm{~A}$, and $18 \% \mathrm{~A}$ after 10 
min of incubation with $10 \mu \mathrm{M}$ 5'-UUGCGG(m6A)CUCCUGUUG-3' (hereafter known as m6A oligo) at $37^{\circ} \mathrm{C}$ (Fig. 1A). Indeed, hm6A persisted (half-life ( $\left.\mathrm{t}_{1 / 2}\right) \sim 3$ hours, SI Appendix, Fig. S2A) as the major product and f6A ( $\mathrm{t}_{1 / 2} \sim 7$ hours, SI Appendix, Fig. S2B) as a minor product, of FTO m6A demethylation over a 60 min time course. In contrast, ALKBH5 $(3.5 \mu \mathrm{M})$ was incubated with $10 \mu \mathrm{M}$ m6A oligo at room temperature and $>98 \%$ demethylation was observed after $10 \mathrm{~min}$ (Fig. 1B). Negligible amounts of $\mathrm{hm} 6 \mathrm{~A}$ and f6A were detected during the monitored time points. We observe a stark contrast between the $\mathrm{m} 6 \mathrm{~A}$ demethylation profiles of FTO and ALKBH5, consistent with previous reports $(23,41)$. FTO behaves like a classical non-heme $\mathrm{Fe}(\mathrm{II})-2 \mathrm{OG}-d e p e n d e n t$ dioxygenase by performing stepwise oxidation, reminiscent of the TET family of enzymes (42), whereas ALKBH5 catalyzes a unique direct m6A-to-A conversion.

ALKBH5 m6A Demethylation Requires One Canonical Iron- and 20G-Dependent Oxidation and Directly Liberates FA. With the observation of direct production of A by ALKBH5, we first sought to decipher how many non-heme $\mathrm{Fe}(\mathrm{II})$ and 2OG-dependent oxidations occur per m6A demethylation by ALKBH5. Because the formation of one non-heme Fe(IV) oxo species consumes one $20 \mathrm{G}$ and produces one succinate molecule, we quantified the amount of succinate converted from $20 \mathrm{O}$ and the corresponding amount of $\mathrm{m} 6 \mathrm{~A}$ and $\mathrm{A}$ produced at 2, 4 and $6 \mathrm{~min}$ in vitro. Importantly, we did not detect significant amounts of hm6A or f6A under these experimental conditions (1.3 $\mu \mathrm{M} \mathrm{ALKBH5,} 40 \mu \mathrm{M} \mathrm{Fe}(\mathrm{II})$, and $40 \mu \mathrm{M} 20 \mathrm{G}$ with $10 \mu \mathrm{M}$ m6A oligo substrate). AlkB enzymes are known to perform non-specific $2 \mathrm{OG}$ turnover in the presence of $\mathrm{Fe}(\mathrm{II})$ and $2 \mathrm{OG}$ in the absence of substrate, and excess succinate is also known to compete with $2 \mathrm{OG}$ for cofactor binding. Various enzymatic conditions were evaluated to ensure that the in vitro reaction was not saturated (e.g., exhibited a linear trend), with less than $25 \%$ of $\mathrm{m} 6 \mathrm{~A}$ consumed over the course of the experiment in order to maximize ALKBH5-m6A engagement and to minimize non-specific $20 \mathrm{G}$ turnover. Succinate was quantified by multiple reaction monitoring (MRM) of the unique mass transition $117 \rightarrow 73.3$ (43) and retention time ( $2 \mathrm{~min})$ (SI Appendix, Fig. S3A and $B)$. A succinate calibration curve showing linearity within the concentration range of succinate generated in vitro was also prepared alongside the same experimental sample to ensure accurate quantification. The corresponding concentrations of $A$ and $m 6 A$ were then quantified using the same set of samples. During the course of this reaction (SI Appendix, Fig. S3C), the rate of succinate production $(0.49$ $\mu \mathrm{M} / \mathrm{min})$ was found to be nearly equal to the rate of $\mathrm{m} 6 \mathrm{~A}$ demethylation $(0.45 \mu \mathrm{M} / \mathrm{min})$ and $A$ production $(0.48 \mu \mathrm{M} / \mathrm{min})$ (Fig. 2A). These data establish that ALKBH5 undergoes one canonical non-heme $\mathrm{Fe}(\mathrm{II})$ 2OG-dependent oxidation per m6A demethylation event.

As the stoichiometry for this ALKBH5-catalyzed m6A demethylation is expected to generate one equivalent of $F A$ per turnover to $A$, we measured the production of $F A$ with respect to $m 6 A$ demethylation and $A$ formation at $0,1,5,10$ and $15 \mathrm{~min}$. Trans-1,2-diaminocyclohexane was used to derivatize FA for quantification via monitoring MRM of the unique mass transition $126.9 \rightarrow 98$ and retention time ( $1.3 \mathrm{~min})(\mathrm{SI}$ Appendix, Fig. S4A-C). A calibration curve was prepared with known concentrations of FA and linearity was ensured within the range of measurement. A control experiment was performed in parallel using control substrate, 5'-UUGCGGACUCCUGUUG-3' (hereafter known as, A oligo) to account for background noise (SI Appendix, Fig. S4D-E). Using this method, we were able to account for ca. $90 \%$ of the FA produced with respect to $\mathrm{m} 6 \mathrm{~A}$ consumed and A formed at each time point after 1 min (Fig. 2B). Notably, the amount of FA detected closely mirrored the formation of A. As FA is a highly reactive carbonyl species, we speculated that the small fraction of unaccounted FA may be consumed by side reactions with the co-substrates and/or buffer components. Nevertheless, these experiments establish that FA is released rapidly and concomitantly upon m6A demethylation by ALKBH5. In contrast, the majority of the single carbon unit for FTO-catalyzed m6A demethylation is trapped in the form of hm6A. In this case, the rate of formaldehyde release by FTO m6A demethylation is an inverse function of the stability of hm6A.

To rule out other possibilities such as a second oxidation of m6A via the intermediacy of hm6A to form f6A, which may liberate formate after hydrolysis, we sought to detect this one-carbon unit. Formate was first derivatized as reported with slight modification (44) (SI Appendix, Fig. S5A and B), quantified using calibration curves prepared with known concentrations of formate, and linearity was ensured within the concentration range of measurement. We performed the experiments using ALKBH5 and a catalytically dead ALKBH5 H204A mutant and measured the amount of formate after 60 min (SI Appendix, Fig. S5C and D). $10 \mu \mathrm{M}$ m6A oligo was $98 \%$ consumed by ALKBH5 while a negligible amount of $\mathrm{m} 6 \mathrm{~A}$ was demethylated by the catalytically dead ALKBH5 H204A mutant, but no detectable amount of formate was measured in either experiment. This negative result is consistent with the aforementioned observations that support a stoichiometry of one non-heme Fe(II)-2OG-dependent oxidation with one 
equivalent of $20 \mathrm{G}$ cofactor consumed and one equivalent of $\mathrm{FA}$ and $\mathrm{A}$ formed per $\mathrm{m} 6 \mathrm{~A}$ oxidative demethylation event catalyzed by ALKBH5.

Active-Site Analysis of FTO and ALKBH5 Provides a Molecular Rationale for the Observed Differences in Product Profiles Upon Oxidative m6A Demethylation. Inspection of the previously reported crystal structure of FTO:m6dA (PDB ID 5ZMD) (39) provides a molecular rationale for why $\mathrm{hm6 \textrm {A }}$ rather than $\mathrm{A}$ is produced as the major product of $\mathrm{m6 \textrm {A }}$ oxidation catalyzed by FTO. Analysis of the structure shows that the m6dA nucleobase forms hydrogen bonds between R96 and the N1 endocyclic nitrogen and between E234 and the N7 endocyclic nitrogen (Fig. 3A). These hydrogen bonds would imbue the nucleobase with a partial positive charge, inductively and mesomerically lowering the $\mathrm{HOMO}$ of the $\mathrm{N} 6$ lone pair and thereby disfavoring Schiff base formation (Fig. 3C), which we posit as a key step in the demethylation mechanism of ALKBH5 (vide infra). ALKBH5, in contrast, has a proline residue (P207) where FTO has E234. Once m6A is oxidized, hm6A may either dissociate from the FTO nucleotide binding region or undergo a second oxidation to produce $66 \mathrm{~A}$ as a minor product, but Schiff base formation is unlikely (Fig. 3C). As attempts to obtain a structure of ALKBH5 complexed to a m6A substrate have yet to prove fruitful, we superimposed the double stranded beta helical (DSBH) domain of the FTO:m6dA complex with the crystal structure of ALKBH5 (PDB ID 4NRP) (41) (SI Appendix, Fig. S6) to probe how ALKBH5 interacts with the m6A nucleobase. The DSBH domains, the conserved catalytic triad (HXD...H motif, where $X$ can be any amino acid), metal ions and $\mathrm{N}$-oxalylglycines (NOG) of ALKBH5 and FTO generally align well ( $\mathrm{rmsd}=4.84 \AA$, by PyMOL) $(\mathrm{SI}$ Appendix, Fig. S6). In the FTO:m6dA complex, V94, R96 and E234 lie in plane with the m6dA nucleobase (Fig. 3A and SI Appendix, Fig. S6). The corresponding residues in ALKBH5 are R130, K132, and P207 respectively (Fig. 3B and SI Appendix, Fig. S6), and these three residues are in plane with the aligned m6dA nucleobase. In addition, V94 and R96 of FTO and R130 and K132 of ALKBH5 are part of the nucleotide recognition loop 1 (NRL1). Together with nucleotide recognition loop 2 (NRL2), these loops are characteristic structural features located at the N-terminal of human AlkB DSBH domain and are known to play a role in substrate recognition $(19,45,46)$. As a hydrophobic residue, the P207 of ALKBH5 is unable to have any polar interactions with the m6dA nucleobase. Instead, we suggest that $\mathrm{m} 6 \mathrm{~A}$ recognition by ALKBH5 is facilitated by hydrogen bond interactions between R130 and the $N 3$ endocyclic nitrogen and K132 and the N1 endocyclic nitrogen (Fig. 3B and SI Appendix, Fig. S6). In addition, we also noticed that Y108 of FTO (Fig. 3A) and Y139 of ALKBH5 (Fig. 3B), both residues within the NRL2, are proximal to the N6-methyl group. This observation led us to propose that the unique m6A demethylation activity of ALKBH5 arises from the interplay of R130, K132, and $\mathrm{Y} 139$ with an hm6A intermediate.

Identification of a Key R130/K132/Y139 Catalytic Triad for ALKBH5-Catalyzed m6A Demethylation. These structural analyses led us to hypothesize that R130, K132, and Y139 play key roles in the mechanism by which ALKBH5 catalyzes m6A demethylation. As a component of the NRL1, $\mathrm{R} 130$ has a clear role in m6A nucleobase recognition. In view of its orientation and proximity, we hypothesized that the proximal basic K132 residue could facilitate dehydration of the N6-hydroxymethyl group formed upon m6A oxidation by serving as a hydrogen bond donor for the hydroxyl leaving group (Fig. 3D). The nascent Schiff base may then be attacked by the now deprotonated, nucleophilic K132 or by $\mathrm{Y} 139$, both of which sit nearby, to form a covalent protein:substrate intermediate. After proton transfer, cleavage of this nascent aminal (for K132) or mixed aminal (for Y139) intermediate to form an iminium or oxocarbenium ion, followed by hydrolysis, would liberate FA.

In order to probe this mechanistic hypothesis, we systematically mutated R130, K132, and $\mathrm{Y} 139$ residues and measured their effects on enzymatic activity and product distribution. The integrity of the mutants' DSBH domain, which houses the catalytic triad (45), was probed using a thermal shift assay (SI Appendix, Fig. S7A-D). For the ALKBH5 wild type protein and mutants, the thermal stability increased (curve shifted right) upon incubation with the generic inhibitor NOG relative to the apo form, indicating that these mutations only perturb the nucleotide recognition domain and not the catalytic iron center. Indeed, the catalytically dead ALKBH5 H204A mutant, which is unable to chelate iron, did not display a thermal shift when incubated with NOG, reflecting NOG specificity in probing the integrity of the catalytic triad (SI Appendix, Fig. S7E).

We incubated $5 \mu \mathrm{M}$ and $10 \mu \mathrm{M}$ ALKBH5 (R130Q) with $10 \mu \mathrm{M}$ of $\mathrm{m} 6 \mathrm{~A}$ oligo for 1 hour at $37^{\circ} \mathrm{C}$ and observed no enzymatic activity (SI Appendix, Fig. S8). In light of its position within the NRL1, we reasoned that the positively charged $\mathrm{R} 130$ is crucial for $\mathrm{m} 6 \mathrm{~A}$ nucleobase recognition through binding via an electrostatic and/or hydrogen bonding interaction with the $N 3$ endocyclic nitrogen lone pair. 
Although Q130 can hydrogen bond to the $N 3$ endocyclic nitrogen, its charge neutral and shorter side chain may attenuate the strength of a hydrogen-bond interaction.

Next, we tested the enzymatic activity and product distribution of ALKBH5 (K132Q). Mutation of K132 to Q132 removes the availability of a relatively acidic ammonium proton near the N6-methyl group but still retains the hydrogen bond interaction capacity, although perhaps weakening it due to a shorter side chain. Interestingly, $5 \mu \mathrm{M}$ ALKBH5 (K132Q) incubated with $10 \mu \mathrm{M} \mathrm{m6A}$ oligo for 1 hour at $37^{\circ} \mathrm{C}$ resulted in the accumulation of 5'-UUGCGG(hm6A)CUCCUGUUG-3' (hereafter known as hm6A oligo) as the major product (Fig. 4A), where ca. $56 \%$ of the $\mathrm{m} 6 \mathrm{~A}$ substrate was oxidized with accumulation of ca. $40 \% \mathrm{hm} 6 \mathrm{~A}, 15 \% \mathrm{~A}$ and $<1 \% \mathrm{f} 6 \mathrm{~A}$ at the 10 -min time point. The amount of $\mathrm{hm} 6 \mathrm{~A}$ peaked at the $30 \mathrm{~min}$ time point at ca. $56 \%$. At the 60 min point, ca. $92 \%$ of $\mathrm{m} 6 \mathrm{~A}$ substrate had been oxidized with accumulation of ca. $55 \% \mathrm{hm6A}, 35 \% \mathrm{~A}$ and $<2 \% \mathrm{f6A}$. The activity of ALKBH5 (K132Q) mutant is more sluggish than its wild-type counterpart and its product profile is similar to that of FTO, with hm6A as the major product. The slower kinetics of $\mathrm{K} 132 \mathrm{Q}$ could be attributed to the lysine residue also playing a role in $\mathrm{m} 6 \mathrm{~A}$ nucleobase recognition and/or substrate positioning for methyl oxidation by affording an electrostatic interaction with the $N 1$ endocyclic nitrogen lone pair (Fig. 3D). Neutral glutamine has a shorter side chain and affords a weaker hydrogen bonding interaction. These data highlight the importance of K132 in the ALKBH5 direct m6A demethylation to A.

Finally, we tested the activity of the ALKBH5 (Y139F) and found that it exhibited low reactivity but produced $A$ as the major product. Negligible amounts of hm6A and f6A were detected. The extent of m6A oxidation was stoichiometric with respect to the input of ALKBH5 (Y139F) after 1 hour at $37^{\circ} \mathrm{C}(\mathrm{SI}$ Appendix, Fig. S9A and B). The hydroxyl group of tyrosine presumably accelerates one or more elementary steps in the demethylation sequence, including the rate-limiting step, since the product profile does not change but the rate of the reaction decreases dramatically with the Y139F mutation. We speculate that Y139 may also affect substrate recognition and/or binding.

Direct Observation of a Covalent Intermediate in ALKBH5-Catalyzed m6A Demethylation. We next posited that the direct demethylation of $\mathrm{m} 6 \mathrm{~A}$ by ALKBH5 via oxidation of $\mathrm{m} 6 \mathrm{~A}$ to $\mathrm{hm} 6 \mathrm{~A}$ might proceed through a covalent intermediate owing to the well-positioned R130/K132/Y139 catalytic triad. In this case, cleavage of the $N 6$ carbon unit by $\mathrm{Fe}(\mathrm{II} / 2-\mathrm{OG}$-catalyzed oxidation could result via a covalent acetal/aminal adduct with participation of K132 and/or Y139, with subsequent hydrolysis liberating the single $\mathrm{N} 6$ carbon of $\mathrm{hm} 6 \mathrm{~A}$ as formaldehyde. This rapid and coordinated direct demethylation mechanism makes it challenging to trap a significant amount of a putative covalent intermediate for detection and visualization. We hypothesized that we might observe a persistent covalent intermediate if we charged active ALKBH5 with $\mathrm{hm6A}$ instead of $\mathrm{m6A}$, reasoning that using this substrate at the next higher oxidation level would result in further oxidation of hm6A to f6A with reformation of $\mathrm{Fe}(\mathrm{II})$ in situ, but that carbon removal from f6A in the presence of $\mathrm{Fe}(\mathrm{II})$ would be slower relative to carbon removal from $\mathrm{hm6A}$ in the presence of $\mathrm{Fe}(\mathrm{II})$, as f6A could form more stable orthoaminal intermediates, with unsaturated or hydrated intermediates also possible due to K132 and/or Y139 interactions (SI Appendix, Fig. S10). Therefore, we incubated active ALKBH5 (loaded with Fe(II) and $2 \mathrm{OG}$ to facilitate formation of the active $\mathrm{Fe}(\mathrm{IV})=\mathrm{O}$ oxidant in situ) directly with $\mathrm{hm} 6 \mathrm{~A}$, anticipating formation of $\mathrm{Fe}(\mathrm{II})$ and f6A and formation of a covalent adduct with $\mathrm{K} 132$ and/or $\mathrm{Y} 139$.

We thus sought to probe for a covalent adduct at the ALKBH5 active site upon incubation with hm6A and designed a native PAGE gel-based assay to detect a potential covalent intermediate (SI Appendix, Fig. S10A). To prepare hm6A, we incubated $5 \mu \mathrm{M}$ FTO with $\sim 10 \mu \mathrm{M} \mathrm{m6A}$ oligo-3'-biotin for $10 \mathrm{~min}$ at $37^{\circ} \mathrm{C}$ to afford $\sim 6 \mu \mathrm{M} \mathrm{hm6A}$ oligo-3'-biotin. We then quenched the reaction by snap freezing it in cryogenic nitrogen and applied freeze-thaw cycles (i.e. warming up to room temperature and then snap freezing) three times, in order to denature the FTO. Without further purification of the reaction mixture, we incubated $2 \mu \mathrm{M}$ ALKBH5 with an estimated final concentration of $3 \mu \mathrm{M} \mathrm{hm6A}$ oligo-3'-biotin at room temperature for one hour. The reaction mixture was then subjected to native PAGE gel electrophoresis at $4^{\circ} \mathrm{C}$. As a negative control, we used A oligo-3'-biotin subjected to the same experimental workflow and conditions. The protein and the nascent covalent intermediate were transferred to PVDF membrane and visualized using multiplex fluorescence imaging.

We expected the covalent intermediate to migrate much faster than its apo ALKBH5 counterpart in a native gel environment due to the higher negative charge to mass ratio imbued by the multi-negatively charged phosphate backbone of the 16-mer RNA oligonucleotide. Indeed, we observed a super-shifted ALKBH5 band in the sample incubated with $\mathrm{hm6A}$ but not with the control sample. The fluorescence 
bands of the super-shifted ALKBH5 and the 3'-biotinylated RNA superimposed perfectly (Fig. 4B). This result confirmed that ALKBH5 forms a covalent adduct with the hm6A oligo-3'-biotin substrate. Moreover, control experiments performed with FTO as well as ALKBH5 R130Q, ALKBH5 K132Q, and ALKBH5 Y139F mutants in the same manner did not give any super-shifted bands in the hm6A and control samples, indicating that these three key residues are required to form the detected covalent intermediate. The faint super-shifted 3'-biotin RNA bands that are present in all lanes are likely experimental artefacts in light of the weak RNA binding affinity of FTO (40).

We performed intact MS experiments on ALKBH5 to further characterize covalent adduct formation. We incubated $2 \mu \mathrm{M}$ ALKBH5 with $~ 3 \mu \mathrm{M}$ final concentration of 5'-UUGCGG(hm6A)CUCCAGAUG-3' (hereafter known as hm6A oligo-2A) and hm6A oligo, respectively. Both hm6A oligos were generated as described above using FTO and the corresponding m6A counterpart. Along with an intense signal for apo ALKBH5 at $28703 \mathrm{Da}$, we identified an intense signal of $33857 \mathrm{Da}$ for hm6A oligo-2A and 33812 $\mathrm{Da}$ for hm6A oligo (SI Appendix, Fig. S11A, B and D), and a difference of 45 Da was measured experimentally. The expected difference between the most intense signals of the two covalent adducts is $46.1 \mathrm{Da}$, based on the difference in MW between two adenosines and two uridines. The experimental value of $45 \mathrm{Da}$ is within a $\pm 1 \mathrm{Da}$ error range of the theoretical value and within the accuracy range of the MS. Furthermore, the deconvoluted peaks of the covalent adducts are separated by 15-16 Da ( $S I$ Appendix, Fig. S11C and E). These additional masses are consistent with incorporation of oxygen atoms into the molecule and may arise via nonspecific oxidations of proximal residues by the nonheme- $\mathrm{Fe}(\mathrm{II})$ center (47). $\mathrm{Fe}(\mathrm{II})$ and $2 \mathrm{OG}$ were introduced together with $\mathrm{hm6 \textrm {A }}$ substrate to facilitate ALKBH5 oxidation of the putative covalent intermediate. Together, these experiments are consistent with detection of a covalent adduct of ALKBH5 and f6A, arising from hm6A oxidation (SI Appendix, Fig. S10B).

Proposed Mechanism for Direct m6A-to-A Conversion Catalyzed by ALKBH5. These observations are consistent with a mechanistic model in which $\mathrm{K} 132$ promotes Schiff base formation on hm6A, which may then undergo subsequent nucleophilic attack by K132 or Y139 (SI Appendix, Fig. S18). The absence of a super-shifted band for ALKBH5 Y139F mutant and its proximity to the nascent iminium upon K132 may alternatively play a role in nucleobase recognition via hydrogen bonding to the N6 nitrogen (Fig. 3D) such that the absence of Y139 disfavours optimal orientation for nucleophilic attack for K132. The absence of a super-shifted band for the K132Q ALKBH5 mutant may be attributed to the inability of this mutant to form a Schiff base and is consistent with the observation of hm6A as the major enzymatic product of $\mathrm{m} 6 \mathrm{~A}$ oxidation. Although it is clear that the interplay between these three residues is required for covalent intermediate formation, we cannot definitively assign which nucleophilic residue initiates the attack on the Schiff base (Fig. 3D). Experiments to probe which residue is the initial nucleophile are complicated by the possibility that a mixed aminal reversibly forms between K132 and Y139 after C-C bond cleavage and prior to release of A (Fig. 5, SI Appendix, Fig. S18), but note that the $\mathrm{K} 132 \mathrm{Q}$ mutant has an altered product distribution whereas the $\mathrm{Y} 139 \mathrm{~F}$ mutant maintains the same product distribution as wildtype is consistent with K132 participating in Schiff base formation.

If K132 were to first perform the nucleophilic attack at the Schiff base, the mechanism would proceed through route I (Fig. 5, SI Appendix, Fig. S18) for adenosine elimination. In the alternative scenario, if Y139 were to first perform the nucleophilic attack at the Schiff base, the mechanism would proceed through route II (Fig. 5, SI Appendix, Fig. S18). Since both intermediates are labile and K132 and Y139 are adjacent to each other in space, formation of a methylene bridge between K132 and Y139 is a probable intermediate prior to hydrolysis and may facilitate release of $A$. In an effort to further probe putative K132 iminium and/or Y139 oxocarbenium intermediates (cf. Fig. 5), we treated ALKBH5 with sodium borohydride $\left(\mathrm{NaBH}_{4}\right)$ mid-reaction with the m6A oligo substrate to trap these intermediates as stable methyl adducts. Indeed, we observed a methyl group on K132 upon digestion and analysis by tandem LC-MS/MS (SI Appendix, Fig. S19). This observation is consistent with covalent bond formation at K132, but cannot exclude the transient involvement of $\mathrm{Y} 139$. Based on our analysis (vide infra) on the position of Y139 on NRL2, the single carbon unit may end up on Y139 prior to release of FA. Although the oxocarbenium arising from Y139 and FA condensation would be less stable, it is also more reactive than the iminium arising from $\mathrm{K} 132$, so the Curtin-Hammett principle may govern the kinetics of FA release. The position of Y139 in NRL2 may also facilitate liberation of formaldehyde. In ALKBH5, K132 lies within a rigid beta strand of NRL1 that forms part of the major beta sheet of the DSBH motif and Y139 lies within the flexible NRL2 region (Fig. 3B and SI Appendix, Fig. S12). Both residues are proximal $(\sim 6.5 \AA$ radius) to the catalytic non-heme $\mathrm{Fe}(\mathrm{II})$ center. This assertion is supported by the observation that the NRL2 (AA 141-148, colored pink) of ALKBH5 (PDB ID 4NRP) is 
disordered in the crystal structure (SI Appendix, Fig. S13), but NRL2s (colored pink) in the crystal structures of AlkB homologs (E. coli AlkB, ALKBH1-3, and FTO) $(39,48-51)$ (SI Appendix, Fig. S13) are not disordered and the amino acid residues are resolved. Furthermore, disordered residue prediction of full length AlkB homologs using IUPred2A server (52), which is based on biophysical models of molecular interactions, supports that the NRL2 region of ALKBH5 has the highest probability of being disordered as compared to other AlkB homologs (SI Appendix, Fig. S14A-F). Therefore, it is possible that the flexible NRL2 could "swing" outward to expose a Y139 oxocarbenium ion/hemiacetal intermediate to the aqueous environment to facilitate hydrolysis and liberation of FA.

ALKBH5 is an Endogenous Source of FA in Live Cells. Finally, we inquired whether direct conversion of $\mathrm{m} 6 \mathrm{~A}$ to $\mathrm{A}$ and FA catalyzed by ALKBH5 serves as a dynamic source of free FA at the cellular level via activity-based sensing of FA fluxes in live cells with genetic perturbation of ALKBH5. Using FAP573 (14), a fluorescent activity-based sensing FA probe developed previously by our laboratory, we compared FA levels in cells with siRNA-mediated knockdown of ALKBH5 (ALKBH5-KD) versus negative control siRNA. The specificity of siALKBH5 was confirmed using western blot analysis (SI Appendix, Fig. S15A and B) and the suitability of anti-ALKBH5 for the immunostaining experiment was verified using a HEK293 (parental line) stably expressing N-terminal myc-tagged ALKBH5. Signal from myc immunostaining and ALKBH5 immunostaining colocalized with DAPI nuclear marker, reflecting ALKBH5 nuclear localization and anti-ALKBH5 specificity (SI Appendix, Fig. S16). HEK293 cells were transfected with $10 \mathrm{nM}$ negative control siRNA and siALKBH5, respectively, and at $\sim 48$ hours post transfection, detection of FA with the FAP573 probe was conducted. As anticipated, FA-dependent FAP573 fluorescence signal intensities (Fig. 6A and B) and levels of ALKBH5 expression (Fig. 6C and $D$ ) in the ALKBH5-KD cells were decreased relative to the control cells. Increasing siRNA doses to 30 nM showed even larger decreases in FAP573 fluorescence signal intensity (Fig. 6E and F) and ALKBH5 knockdown (Fig. $6 \mathrm{G}$ and $\mathrm{H}$ ). Analogous experiments were performed in the MCF-7 breast cancer cell line since ALKBH5 is known to play a role in stem cell proliferation in breast cancer (27). MCF-7 cells were transfected with $30 \mathrm{nM}$ of negative scrambled control siRNA or siALKBH5, and as was observed in the HEK cell models, FA-dependent FAP573 fluorescence signal intensity (Fig. $6 \mathrm{I}$ and $\mathrm{J}$ ) and ALKBH5-dependent immunostaining (Fig. $6 \mathrm{~K}$ and $\mathrm{L}$ ) levels were lower in ALKBH5-KD cells relative to negative control siRNA. Taken together, these experiments establish that ALKBH5 expression, which decreases m6A levels in cells by erasing one-carbon RNA marks (33), also affects the amount of free FA production as this non-heme iron enzyme produces one equivalent of FA per demethylation event.

\section{Concluding Remarks}

We have identified a molecular basis for distinct pathways for oxidative $N$-demethylation of RNA catalyzed by the non-heme iron enzymes FTO and ALKBH5 involved in epigenetic regulation that promote varying release rates of formaldehyde, a potent genotoxin that is endogenously produced in significant amounts in cells. We discovered that ALKBH5 directly demethylates m6A to $A$ with concomitant and rapid FA formation, whereas FTO gives $\mathrm{hm6A}$ as a major product (Fig. 1A) and f6A as a minor product. The $t_{1 / 2}$ of hm6A ( 3 hours) produced by FTO leads to FA release over longer timescales. The collective data are consistent with a model in which ALKBH5 catalyzes m6A-to-A conversion by an unprecedented covalent intermediate mediated by a key R130/K132/Y139 catalytic triad, which is conserved among human, mouse, and zebrafish (SI Appendix, Fig. S17), with rapid formation of both $A$ and $F A$.

This work provides further foundational evidence for non-redundant roles of these major RNA demethylase eraser enzymes that have broader biological implications. Indeed, emerging data show that FTO and ALKBH5 exhibit distinct substrate preferences, including different sites on mRNA, tRNA, and small nuclear RNAs $(4,27,40,53,54)$, with FTO having a broader substrate preference (m6A, hm6A, N1-methyladenosine (m1A) and N6,2'-O-dimethyladenosine (m6Am)) whereas ALKBH5 is more specific towards m6A in mRNA. Such differences have spawned a field of RNA epigenetics. Our findings point to differences in FA production as another factor that distinguishes these classes of enzymes, as the rate of FA released into the cellular environment depends on the stability of the $\mathrm{N}$ hydroxymethylated intermediate (hm6A). The distinct m6A demethylation mechanisms of FTO and ALKBH5 can affect the spatiotemporal dynamics of FA availability in cells. In the case of ALKBH5, the single carbon unit in $\mathrm{m6A}$ can be discharged rapidly as FA to the immediate environment. For FTO, FA is discharged/released slowly and at a distance proportional to diffusion of hm6A away from the location of $\mathrm{m} 6 \mathrm{~A}$ demethylation, since the one-carbon unit is released in the form of stable hm6A product. Taking into account $\mathrm{N}$-hydroxymethylated nucleobases more broadly, it has been shown that the stability can range from hours (hm6A) to seconds (N3-hydroxymethylthymidine (hm3T) and N3- 
hydroxylmethyluridine (hm3U)) under physiological conditions (22), which can in turn be potential cellular sources of FA. Moreover, unlike lysine demethylation in histones catalyzed by lysine-specific histone demethylase 1 (LSD1), where FA is sequestered by the co-substrate tetrahydrofolate (THF) upon release (55), it is tempting to speculate that ALKBH5 has evolved to purposefully release FA for possible biological regulatory purposes upon $\mathrm{m} 6 \mathrm{~A}$ demethylation. Indeed, the cellular imaging data (Fig. 6) show that free FA levels are modulated in an ALKBH5-dependent manner. We hypothesize that the spatiotemporal availability of FA, a potent one-carbon electrophile, can influence proximal protein and nucleic acid targets and give rise to a continuum between one-carbon metabolism and one-carbon signaling.

\section{Materials and Methods}

Details of reagents and experimental procedures for cDNA sequences, cloning, bacteria and mammalian cells expression and purification of wild type FTO, ALKBH5 and ALKBH5 mutants, f6A synthesis, concise methods for $\mathrm{m} 6 \mathrm{~A}, \mathrm{hm} 6 \mathrm{~A}, \mathrm{f} 6 \mathrm{~A}$ and $\mathrm{A}$ quantification, in vitro enzymatic assays, formaldehyde quantification, formate quantification, succinate quantification, native gel cross-linking assay, intact MS assays, thermal shift assays, cell culture, stable cell lines generation, western blot, siRNA knockdown, immunofluorescence and live cell imaging experiments, and statistical analysis are described in the SI Appendix.

\section{Acknowledgements}

We thank the NIH (ES28096 and ES4705 to C.J.C.) for research support. J.D.W.T. thanks A*STAR (Singapore) for a postdoctoral fellowship. S.W.M.C. was supported by the AGBT-Elaine R. Mardis Fellowship in Cancer Genomics from the Damon Runyon Cancer Research Foundation and The Genome Partnership, Inc. (DRG-2395-20). K.J.B. thanks the National Science Foundation for a graduate fellowship. D.H. was supported as a Tang Foundation Distinguished Scholar (UC Berkeley QB3). D.A.I. was supported by the Howard Hughes Medical Institute as a Life Science Research Foundation Post Fellow. We thank Alison Killilea, Carissa Tasto, and Molly Fischer of the UC Berkeley Cell Culture Facility for cell culture support and Dr. Anthony lavarone of the QB3 mass spectrometry facility for proteomics support. We thank Douglas Miller, Monica Neugebauer, Kersh Thevasundaram and Prof. Michelle Chang for use of and technical assistance with their Agilent 6460 UPLC-Triple Quadrupole-MS/MS.

\section{References:}

1. G. S. Ducker, J. D. Rabinowitz, One-Carbon Metabolism in Health and Disease. Cell Metab 25, 27-42 (2017).

2. J. W. Locasale, Serine, glycine and one-carbon units: cancer metabolism in full circle. Nat Rev Cancer 13, 572-583 (2013).

3. Y. Shi et al., Histone demethylation mediated by the nuclear amine oxidase homolog LSD1. Cell 119, 941-953 (2004).

4. G. Jia et al., N6-methyladenosine in nuclear RNA is a major substrate of the obesityassociated FTO. Nat Chem Biol 7, 885-887 (2011).

5. L. B. Pontel et al., Endogenous Formaldehyde Is a Hematopoietic Stem Cell Genotoxin and Metabolic Carcinogen. Mol Cell 60, 177-188 (2015).

6. G. Burgos-Barragan et al., Mammals divert endogenous genotoxic formaldehyde into onecarbon metabolism. Nature 548, 549-554 (2017).

7. K. J. Bruemmer, T. F. Brewer, C. J. Chang, Fluorescent probes for imaging formaldehyde in biological systems. Curr Opin Chem Biol 39, 17-23 (2017).

8. J. Ohata, K. J. Bruemmer, C. J. Chang, Activity-Based Sensing Methods for Monitoring the Reactive Carbon Species Carbon Monoxide and Formaldehyde in Living Systems. Acc Chem Res 52, 2841-2848 (2019).

9. T. F. Brewer, C. J. Chang, An Aza-Cope Reactivity-Based Fluorescent Probe for Imaging Formaldehyde in Living Cells. J Am Chem Soc 137, 10886-10889 (2015).

10. A. Roth, H. Li, C. Anorma, J. Chan, A Reaction-Based Fluorescent Probe for Imaging of Formaldehyde in Living Cells. J Am Chem Soc 137, 10890-10893 (2015).

11. W. Liu et al., A reactivity-based [(18)F]FDG probe for in vivo formaldehyde imaging using positron emission tomography. Chem Sci 7, 5503-5507 (2016).

12. Y. Tang, X. Kong, A. Xu, B. Dong, W. Lin, Development of a Two-Photon Fluorescent Probe for Imaging of Endogenous Formaldehyde in Living Tissues. Angew Chem Int Ed Engl 55, 3356-3359 (2016). 
13. T. F. Brewer, G. Burgos-Barragan, N. Wit, K. J. Patel, C. J. Chang, A 2-aza-Cope reactivitybased platform for ratiometric fluorescence imaging of formaldehyde in living cells. Chem Sci 8, 4073-4081 (2017).

14. K. J. Bruemmer et al., Development of a General Aza-Cope Reaction Trigger Applied to Fluorescence Imaging of Formaldehyde in Living Cells. J Am Chem Soc 139, 5338-5350 (2017).

15. K. J. Bruemmer, O. Green, T. A. Su, D. Shabat, C. J. Chang, Chemiluminescent Probes for Activity-Based Sensing of Formaldehyde Released from Folate Degradation in Living Mice. Angew Chem Int Ed Engl 57, 7508-7512 (2018).

16. A. S. Tibbetts, D. R. Appling, Compartmentalization of Mammalian folate-mediated onecarbon metabolism. Annu Rev Nutr 30, 57-81 (2010).

17. T. Gerken et al., The obesity-associated FTO gene encodes a 2-oxoglutarate-dependent nucleic acid demethylase. Science 318, 1469-1472 (2007).

18. C. Yi, C. G. Yang, C. He, A non-heme iron-mediated chemical demethylation in DNA and RNA. Acc Chem Res 42, 519-529 (2009).

19. L. J. Walport, R. J. Hopkinson, C. J. Schofield, Mechanisms of human histone and nucleic acid demethylases. Curr Opin Chem Biol 16, 525-534 (2012).

20. C. Q. Herr, R. P. Hausinger, Amazing Diversity in Biochemical Roles of Fe(II)/2-Oxoglutarate Oxygenases. Trends Biochem Sci 43, 517-532 (2018).

21. R. J. Klose, E. M. Kallin, Y. Zhang, JmjC-domain-containing proteins and histone demethylation. Nature Reviews Genetics 7, 715-727 (2006).

22. S. Shishodia et al., NMR analyses on N-hydroxymethylated nucleobases - implications for formaldehyde toxicity and nucleic acid demethylases. Org Biomol Chem 16, 4021-4032 (2018).

23. Y. Fu et al., FTO-mediated formation of N6-hydroxymethyladenosine and N6formyladenosine in mammalian RNA. Nat Commun 4, 1798 (2013).

24. D. Dominissini et al., Topology of the human and mouse m6A RNA methylomes revealed by m6A-seq. Nature 485, 201-206 (2012).

25. K. D. Meyer et al., Comprehensive analysis of mRNA methylation reveals enrichment in 3' UTRs and near stop codons. Cell 149, 1635-1646 (2012).

26. J. Liu et al., A METTL3-METTL14 complex mediates mammalian nuclear RNA N6-adenosine methylation. Nat Chem Biol 10, 93-95 (2014).

27. G. Zheng et al., ALKBH5 is a mammalian RNA demethylase that impacts RNA metabolism and mouse fertility. Mol Cell 49, 18-29 (2013).

28. X. Wang et al., N6-methyladenosine-dependent regulation of messenger RNA stability. Nature 505, 117-120 (2014).

29. X. Zhao et al., FTO-dependent demethylation of N6-methyladenosine regulates mRNA splicing and is required for adipogenesis. Cell Res 24, 1403-1419 (2014).

30. K. D. Meyer et al., 5' UTR m(6)A Promotes Cap-Independent Translation. Cell 163, 999-1010 (2015).

31. H. Shi et al., YTHDF3 facilitates translation and decay of $\mathrm{N}(6)$-methyladenosine-modified RNA. Cell Res 27, 315-328 (2017).

32. Y. Liu et al., N (6)-methyladenosine RNA modification-mediated cellular metabolism rewiring inhibits viral replication. Science 365, 1171-1176 (2019).

33. C. Zhang et al., Hypoxia induces the breast cancer stem cell phenotype by HIF-dependent and ALKBH5-mediated m6-demethylation of NANOG mRNA. Proc Natl Acad Sci U S A 113, E2047-2056 (2016).

34. Z. Li et al., FTO Plays an Oncogenic Role in Acute Myeloid Leukemia as a N(6)Methyladenosine RNA Demethylase. Cancer Cell 31, 127-141 (2017).

35. S. Zhang et al., m(6)A Demethylase ALKBH5 Maintains Tumorigenicity of Glioblastoma Stem-like Cells by Sustaining FOXM1 Expression and Cell Proliferation Program. Cancer Cell 31, 591-606.e596 (2017).

36. R. Su et al., Targeting FTO Suppresses Cancer Stem Cell Maintenance and Immune Evasion. Cancer Cell 38, 79-96.e11 (2020).

37. H. Shi, J. Wei, C. He, Where, When, and How: Context-Dependent Functions of RNA Methylation Writers, Readers, and Erasers. Mol Cell 74, 640-650 (2019).

38. S. Zaccara, R. J. Ries, S. R. Jaffrey, Reading, writing and erasing mRNA methylation. Nat Rev Mol Cell Biol 20, 608-624 (2019).

39. X. Zhang et al., Structural insights into FTO's catalytic mechanism for the demethylation of multiple RNA substrates. Proc Natl Acad Sci U S A 116, 2919-2924 (2019). 
40. J. Wei et al., Differential $m(6) A, m(6) A(m)$, and $m(1) A$ Demethylation Mediated by FTO in the Cell Nucleus and Cytoplasm. Mol Cell 71, 973-985.e975 (2018).

41. C. Feng et al., Crystal structures of the human RNA demethylase Alkbh5 reveal basis for substrate recognition. J Biol Chem 289, 11571-11583 (2014).

42. L. Hu et al., Structural insight into substrate preference for TET-mediated oxidation. Nature 527, 118-122 (2015).

43. O. Al Kadhi, A. Melchini, R. Mithen, S. Saha, Development of a LC-MS/MS Method for the Simultaneous Detection of Tricarboxylic Acid Cycle Intermediates in a Range of Biological Matrices. J Anal Methods Chem 2017, 5391832 (2017).

44. D. M. Warui et al., Detection of formate, rather than carbon monoxide, as the stoichiometric coproduct in conversion of fatty aldehydes to alkanes by a cyanobacterial aldehyde decarbonylase. J Am Chem Soc 133, 3316-3319 (2011).

45. M. A. McDonough, C. Loenarz, R. Chowdhury, I. J. Clifton, C. J. Schofield, Structural studies on human 2-oxoglutarate dependent oxygenases. Curr Opin Struct Biol 20, 659-672 (2010).

46. J. D. W. Toh et al., A strategy based on nucleotide specificity leads to a subfamily-selective and cell-active inhibitor of N(6)-methyladenosine demethylase FTO. Chem Sci 6, 112-122 (2015).

47. J. M. Bollinger, Jr., C. Krebs, Enzymatic C-H activation by metal-superoxo intermediates. Curr Opin Chem Biol 11, 151-158 (2007).

48. O. Sundheim et al., Human $\mathrm{ABH} 3$ structure and key residues for oxidative demethylation to reverse DNA/RNA damage. Embo $j$ 25, 3389-3397 (2006).

49. C. G. Yang et al., Crystal structures of DNA/RNA repair enzymes AlkB and ABH2 bound to dsDNA. Nature 452, 961-965 (2008).

50. C. Zhu, C. Yi, Switching demethylation activities between AlkB family RNA/DNA demethylases through exchange of active-site residues. Angew Chem Int Ed Engl 53, 36593662 (2014).

51. L. F. Tian et al., Structural basis of nucleic acid recognition and $6 \mathrm{~mA}$ demethylation by human ALKBH1. Cell Res 30, 272-275 (2020).

52. B. Mészáros, G. Erdos, Z. Dosztányi, IUPred2A: context-dependent prediction of protein disorder as a function of redox state and protein binding. Nucleic Acids Res 46, W329-w337 (2018).

53. J. Mauer et al., Reversible methylation of $\mathrm{m}(6) \mathrm{A}(\mathrm{m})$ in the $5^{\prime}$ cap controls mRNA stability. Nature 541, 371-375 (2017).

54. J. Mauer et al., FTO controls reversible m(6)Am RNA methylation during snRNA biogenesis. Nat Chem Biol 15, 340-347 (2019).

55. Z. Luka, F. Moss, L. V. Loukachevitch, D. J. Bornhop, C. Wagner, Histone demethylase LSD1 is a folate-binding protein. Biochemistry 50, 4750-4756 (2011). 


\section{Figures and Legends}
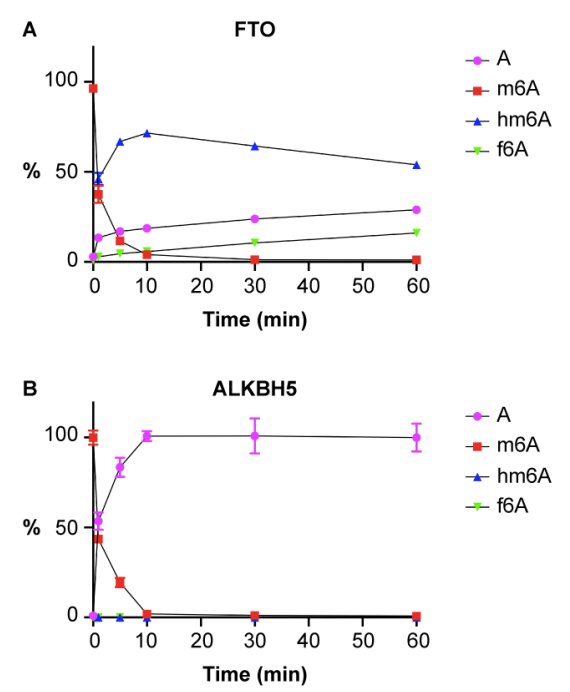

Fig. 1. Time course profile of m6A demethylation by (A) FTO and (B) ALKBH5. Products formed at various time points $(0,1,5,10,30$ and $60 \mathrm{~min})$ where (A) $5 \mu \mathrm{M}$ FTO and (B) $3.5 \mu \mathrm{M}$ ALKBH5 were incubated with $10 \mu \mathrm{M}$ of $\mathrm{m} 6 \mathrm{~A}$ oligo at $37^{\circ} \mathrm{C}$ and room temperature respectively. The individual concentrations of $\mathrm{A}, \mathrm{m} 6 \mathrm{~A}, \mathrm{hm} 6 \mathrm{~A}$, and f6A quantified at each time point were expressed as percentage (\%, Y-axis), where the sum of the concentrations of $\mathrm{A}, \mathrm{m} 6 \mathrm{~A}, \mathrm{hm} 6 \mathrm{~A}$, and f6A at each time points adds up to $100 \%$. Error bars indicate the mean $\pm S D$ ( $n=3$ biological replicates).
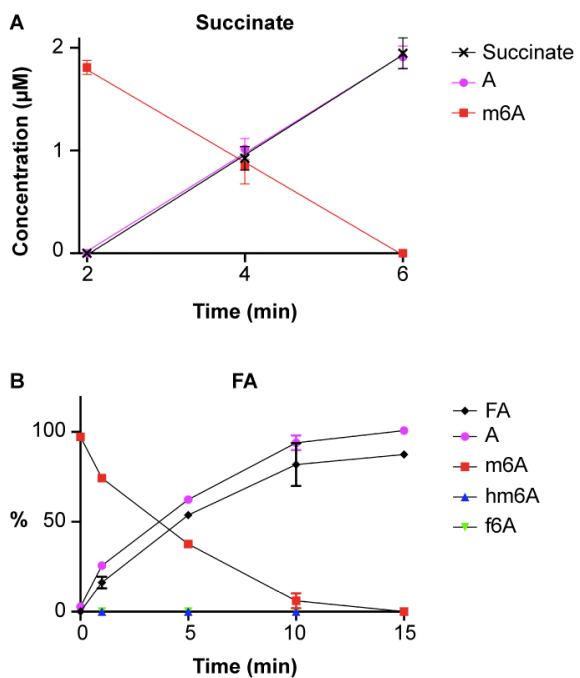

Fig. 2. Characterization of stoichiometry of ALKBH5-catalyzed m6A demethylation. (A) Quantification of succinate and adenosine produced per m6A demethylation event at 2, 4 and 6 min of reaction. $Y$ axis (concentration $(\mu \mathrm{M})$ ) represents the changes in concentrations of $\mathrm{m} 6 \mathrm{~A}, \mathrm{~A}$ and succinate across 2 , 4 and $6 \mathrm{~min}$ time points. Over the course of 2 to $6 \mathrm{~min}$ in vitro reaction time points monitored, the concentration of m6A decreased in a linear manner by $\sim 1.8 \mu \mathrm{M}$, and both concentrations of succinate and $\mathrm{A}$ increased in a linear manner by $\sim 1.9 \mu \mathrm{M}$. Reaction conditions: $1.3 \mu \mathrm{M}$ ALKBH5 was incubated with $40 \mu \mathrm{M} \mathrm{Fe}(\mathrm{II}), 40 \mu \mathrm{M} \mathrm{2OG}$, and $10 \mu \mathrm{M}$ m6A oligo at room temperature. The rate of succinate produced $(\sim 0.487 \mu \mathrm{M} / \mathrm{min})$ is nearly equal to the rate of $\mathrm{m} 6 \mathrm{~A}$ demethylation $(\sim 0.451 \mu \mathrm{M} / \mathrm{min})$ and $\mathrm{A}$ production $(\sim 0.478 \mu \mathrm{M} / \mathrm{min})$. Error bars indicate the mean $\pm \mathrm{SD}(n=4,2$ biological replicates $X 2$ technical replicates). (B) In vitro time-course tracking of formaldehyde (FA) produced over the monitored time points $(0,1,5,10$ and $15 \mathrm{~min})$. The amount of $F A$ is quantified and presented as a percentage (Y-axis) of the sum of the concentrations of $A, m 6 A, h m 6 A$ and $f 6 A$ at each time point. Error bars indicate the mean $\pm S D$ ( $n=3$ biological replicates). 


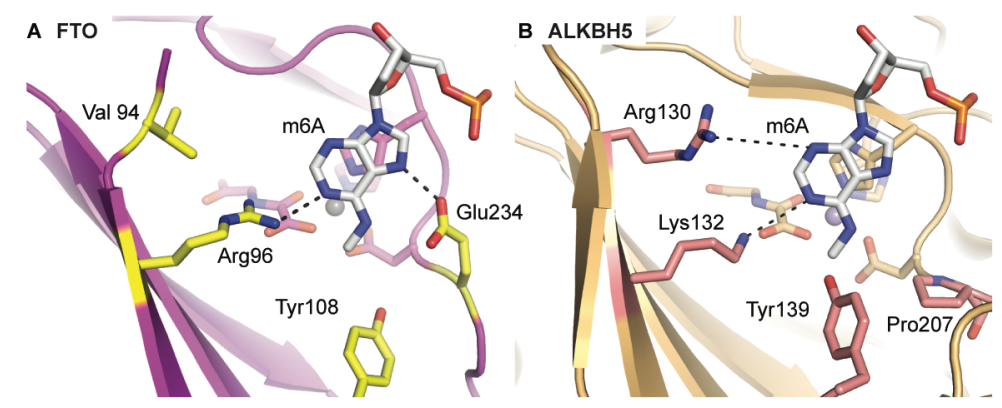

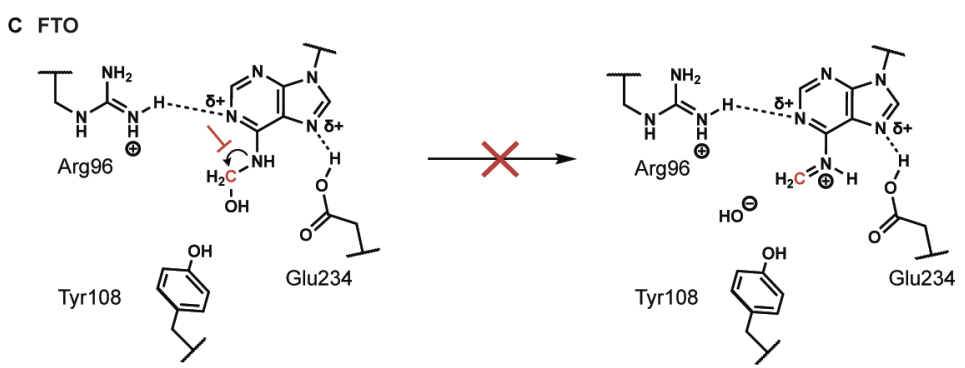

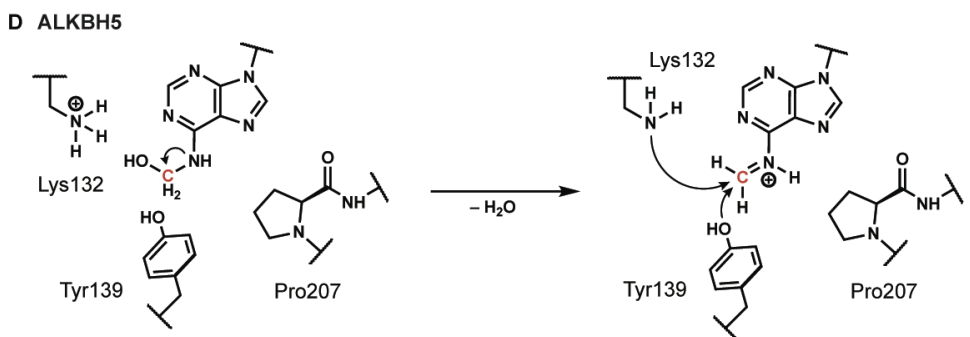

Fig. 3. Structural analysis of FTO and ALKBH5 provides insight into m6A demethylation biochemistry. (A) Nucleobase recognition region of FTO:m6dA co-crystal structure (PDB ID 5ZMD, purple). Key residues which facilitate understanding of the FTO m6A demethylation mechanism are in yellow sticks. (B) Superimposition of ALKBH5 (PDB ID 4NRP, pale orange) with FTO:m6dA co-crystal structure, with FTO removed for clarity. Key residues which facilitate understanding of the unique m6A demethylation mechanism of ALKBH5 are in pink sticks. (C) A graphical illustration of the FTO nucleobase recognition region interacting with $\mathrm{hm6A}$. An additional hydrogen bond with E234 should disfavor Schiff base formation and explains why hm6A is the major product of FTO. (D) A graphical illustration of how K132 and $\mathrm{Y} 139$ of ALKBH5 in the nucleobase recognition region facilitate formation of a covalent intermediate via elimination of water. 

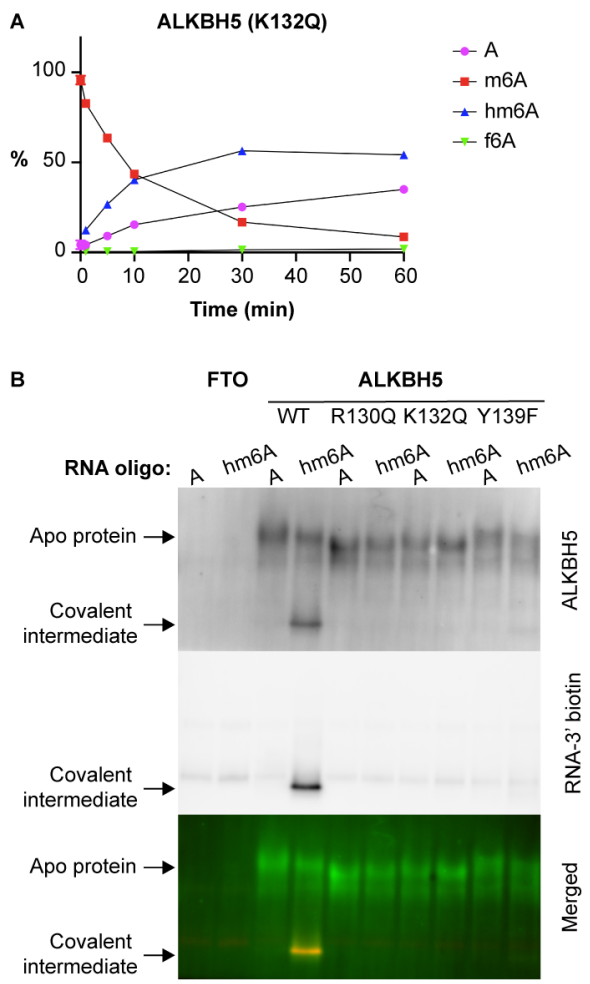

Fig. 4. Identification and characterization of a covalent intermediate in $m 6 \mathrm{~A}$ demethylation catalyzed by ALKBH5. (A) Time course profile of $10 \mu \mathrm{M}$ m6A oligo incubated with $5 \mu \mathrm{M}$ ALKBH5 (K132Q) at $37^{\circ} \mathrm{C}$. Products formed at various time points $(0,1,5,10,30$ and $60 \mathrm{~min})$ were monitored. The individual concentrations of $\mathrm{A}, \mathrm{m} 6 \mathrm{~A}, \mathrm{hm} 6 \mathrm{~A}$, and $\mathrm{f} 6 \mathrm{~A}$ quantified at each time point were expressed as a percentage $(\%, Y$-axis) where the sum of the concentrations of $A, m 6 A, h m 6 A$, and $f 6 A$ at each time point adds up to $100 \%$. Error bars indicate the mean \pm SD ( $n=3$ biological replicates). (B) Native PAGE gel assay to visualize the covalent intermediate via multiplex fluorescent imaging. Representative runs included ALKBH5 and control proteins (FTO and three ALKBH5 mutants). RNA oligomers are 3' biotinylated for tracking and imaging. ALKBH5 was imaged using primary anti-ALKBH5 (rabbit) with a secondary antibody Alexa Fluor 546. 3'-biotinylated RNA oligos were read out using a streptavidin conjugated to Alexa Fluor 647.

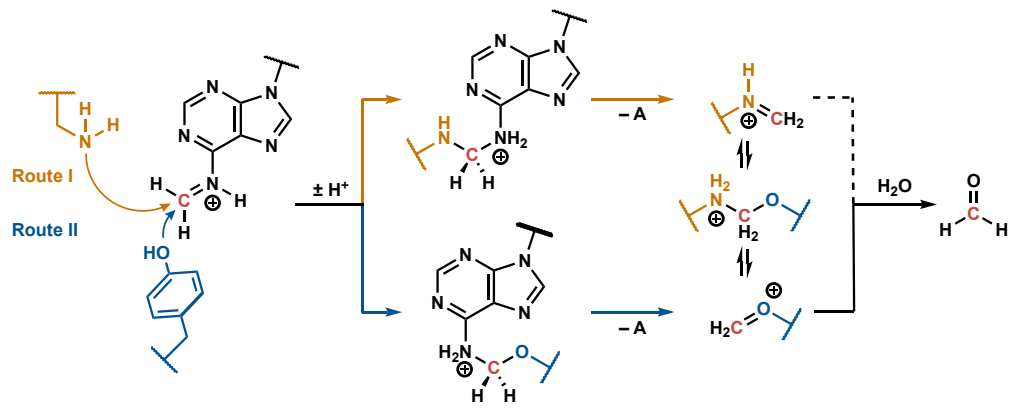

Fig. 5. Proposed mechanistic pathways that lead to fast release of FA catalyzed by ALKBH5. Route I (orange), where K132 attacks the Schiff base. Route II (blue), where Y139 attacks the Schiff base. Elimination of adenosine (A) via Route I yields an iminium while via Route II yields an oxocarbenium. Intermediate formation of a methylene bridge is probable, as is transfer of the single carbon unit (red) to Y139 for eventual formaldehyde release. Note: All steps are chemically reversible, but reactions are depicted in the forward direction for simplicity. 

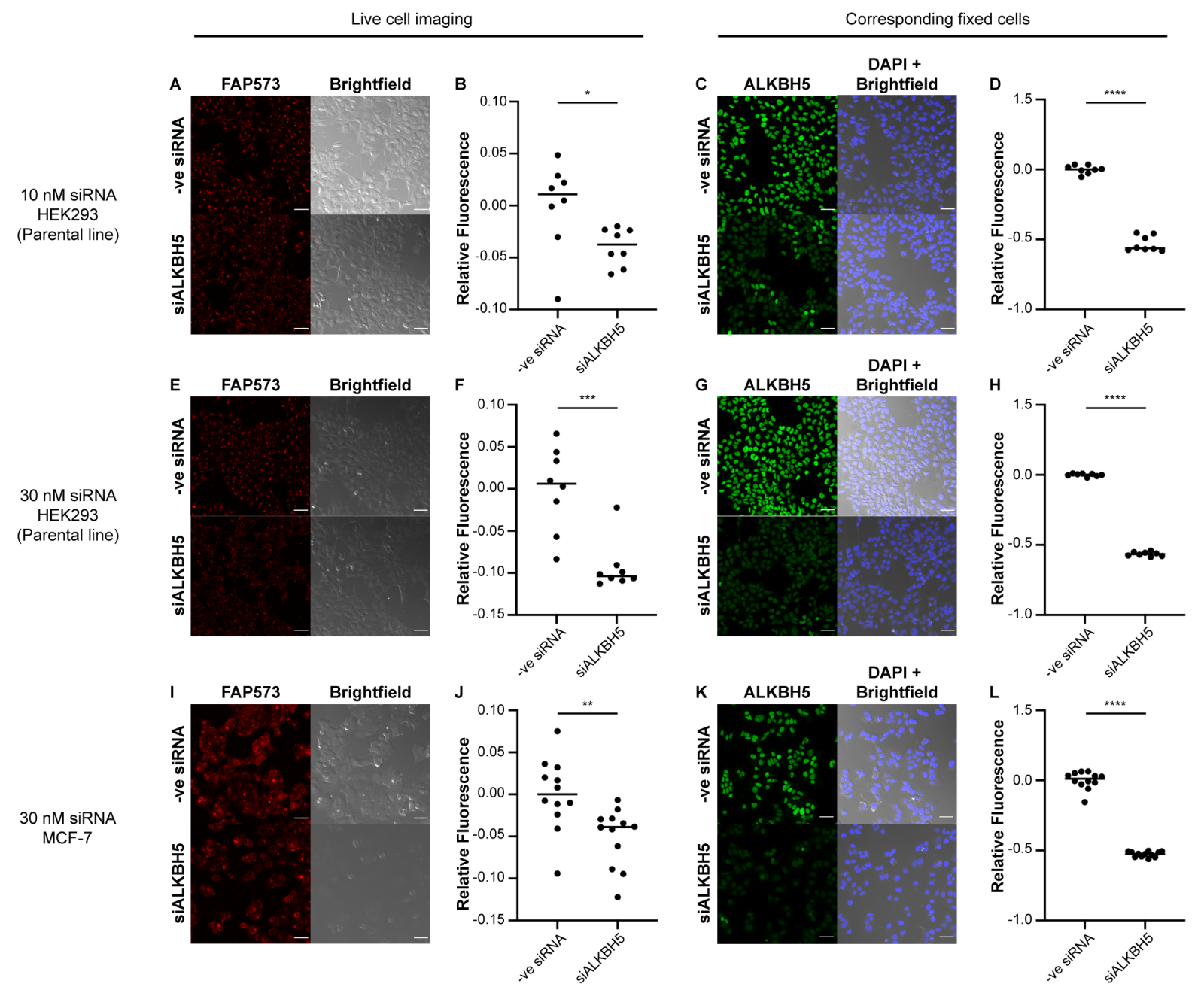

Fig. 6. Activity-based sensing with FAP573 shows ALKBH5-dependent FA production in live cells. (A, $\mathrm{B}, \mathrm{C}$ and D) ALKBH5 KD in HEK293 (parental line) transfected with $10 \mathrm{nM}$ siRNA. (E, F, G and H) ALKBH5 KD in HEK293 (parental line) transfected with $30 \mathrm{nM}$ siRNA. (I, J, K and L) ALKBH5 KD in MCF-7 cell line transfected with $30 \mathrm{nM}$ siRNA. (A, E and I) Live cell imaging of FAP573 fluorescence was performed $\sim 48$ hours post-transfection of negative control siRNA (-ve siRNA, top row) and siALKBH5 (bottom row). FAP573 fluorescence (left column) and corresponding brightfield images (right column). ( $B$ and $F$ ) Data of fluorescence intensities from 4 wells $X 2$ regions of interest (ROIs) per well $(n=8)$. Horizontal bar is the average of the 8 data points. For data presentation, FAP573 fluorescence data points for -ve siRNA is set to 0 and FAP573 fluorescence data points for siALKBH5 is presented as an offset from $0\left({ }^{*} \mathrm{p}<0.0315,{ }^{* * *} \mathrm{p}<0.0005\right)$. (C, G and $\left.\mathrm{K}\right)$ Live cells used for FAP573 experiment were fixed for ALKBH5 immunostaining. -ve siRNA (top row) and siALKBH5 (bottom row). ALKBH5 immunofluorescence (left column) and corresponding DAPI + brightfield images (right column). (D and H) Data of fluorescence intensities from 4 wells $X 2$ ROls per well $(n=8)$. Horizontal bar is the average of the 8 data points. For data presentation, ALKBH5 immunofluorescence data points for -ve siRNA is set to 0 and ALKBH5 immunofluorescence data points for siALKBH5 is presented as an offset from 0 $\left({ }^{* * * *} p<0.0001\right)$. (J) Data of fluorescence intensities from 4 wells $X 3$ ROls per well $(n=12)$. Horizontal bar is the average of the 12 data points. For data presentation, FAP573 fluorescence data points for ve siRNA is set to 0 and FAP573 fluorescence data points for siALKBH5 is presented as an offset from $0\left(^{* *} p<0.0045\right)$. (L) Data of fluorescence intensities from 4 wells $X 3$ ROls per well $(n=12)$. Horizontal bar is the average of the 12 data points. For data presentation, ALKBH5 immunofluorescence data points -ve siRNA is set to 0 and ALKBH5 immunofluorescence data points for siALKBH5 is presented as an offset from $0\left({ }^{* * *} \mathrm{p}<0.0001\right)$. Scale bar represents $50 \mu \mathrm{m}$ in all images. 